\title{
A Low-Cost Distributed Instrumentation System for Monitoring, Identifying and Diagnosing Irregular Patterns of Behavior in Critical ITS Components
}

\author{
Ekasit Vorakitolan ${ }^{1}$, Nick Mould ${ }^{1}$, Joseph P. Havlicek ${ }^{1}$, Ronald D. Barnes ${ }^{1}$, and Alan R. Stevenson ${ }^{2}$
}

\begin{abstract}
ITS telecommunication infrastructure and information gathering/distribution equipment such as fiber-optic routing devices, communication huts, uninterrupted power supplies (UPSs), cameras and dynamic message signs (DMSs) are critical to the effective management of resources in emergency situations. Frequently encountered scenarios include evaluating the severity of vehicle collisions to dispatch appropriate law enforcement and ambulatory services, or issuing time-sensitive AMBER alerts to assist in the effort to find missing or kidnapped children. Due to the unacceptably high cost of ITS equipment failures, preventative maintenance and dense operational testing are high priorities. In this paper we present a low-cost distributed instrumentation system (DIS) for continuous monitoring of critical ITS components. Over the last three years, we have developed and deployed the DIS in the Oklahoma Department of Transportation (ODOT) private fiberoptic network that spans several major metropolitan areas in and around Oklahoma City, Tulsa and Lawton. The Oklahoma DIS is responsible for monitoring the health of the entire ODOT ITS communications network as well as the integrity of each camera video signal and the operational status of each DMS. All of the information acquired by the DIS is integrated into an operational summary that is available on a private website for the design and execution of ITS equipment maintenance plans. In Oklahoma, information acquired by the DIS has been successfully integrated into a wide range of operation and maintenance (O\&M) planning, which has led to a significant improvement in terms of overall ITS quality of service $(Q 0 S)$ and a quantifiable reduction in wasted costs associated with the premature discarding of energy storage devices.
\end{abstract}

\section{INTRODUCTION}

Intelligent transportation system (ITS) equipment such as Dynamic message sings (DMSs), cameras and traffic speed sensors are responsible for providing a wide range of critical information to the public, such as description(s) of missing children and their captors in AMBER alert situations, evacuation and safety instructions in the case of severe weather (tornadoes, hurricanes), roadway weather information (ice, snow) and road closure information (floods, wild fires). Since its inception in 1997, the AMBER alert system has contributed to the recovery of many lost or abducted children through instrumental application of several ITS technologies. With a total of 174,000 reports of missing children since

\footnotetext{
${ }^{1}$ Ekasit Vorakitolan, Nick Mould, Joseph P. Havlicek and Ronald D. Barnes are with the University of Oklahoma School of Electrical and Computer Engineering, 110 W. Boyd Street, Devon Energy Hall 150, Norman, OK, USA 73019-1102. Their email addresses are respectively, ekasitv@ou.edu, stupid@ou.edu, joebob@ou.edu and ron@ou.edu.

${ }^{2}$ Alan R. Stevenson is with the the Oklahoma Department of Transportation, Technology Services Division, Oklahoma City, OK, USA 73105. His email address is astevenson@odot.org.

This work was supported in part by grants from the Oklahoma Department of Transportation and the Oklahoma Highway Safety Office.
}

1984 and a relatively steady 200 to 300 AMBER alerts issued per year, the importance of improving the reliability of ITS field equipment cannot be overstated [1]-[5].

A wide variety of established operations and maintenance (O\&M) plans that are based on combinations of preventative and corrective maintenance techniques have been reported within the ITS literature [6]-[9]. At a relatively high cost, the implementation of elaborate O\&M plans has been demonstrably verified as an effective method for significantly reducing the probability of equipment failure, length of system downtime and risk of service interruption [10]. As a consequence, planning and execution of comprehensive $\mathrm{O} \& \mathrm{M}$ procedures is a major component in many strategic ITS plans [6], [7], [11].

The cost of ITS O\&M plans varies based on the size of the geographic area spanned by the ITS equipment, the diversity of the equipment and the total number of pieces of equipment. In 1997, the Texas DOT suggested that O\&M should cost between $10 \%$ and $15 \%$ of the ITS capital fund, resulting in an estimated maintenance cost of 8.7 million USD for the State of Texas [11]. In 2004, the Florida DOT established a ten year ITS plan with an estimated O\&M budget of approximately 142 million USD or 14.2 million USD per year [6]. With respect to a per unit cost estimate, the Oregon DOT has spent 1 million USD annually to support 1,200 devices, while the California Transportation Department spends approximately 2.6 million USD per year for 2,212 devices [9].

In many states, implementation of elaborate operation and maintenance $(\mathrm{O} \& \mathrm{M})$ plans are not possible because of the high cost associated with dispatching large numbers of engineers and technicians to perform preventative maintenance and testing on expensive ITS equipment [7], [8]. Popular low-cost O\&M strategies include dependence on manufacturer suggested mean time before failure (MTBF) estimates for maintenance scheduling, adoption of vendor specific native device monitoring hardware/software solutions and utilization of primitive diagnostic tools such as the simple network monitoring protocol (SNMP), all of which are widely available within most ITS devices. In general, manufacturer specific maintenance or device monitoring solutions suffer from extremely conservative MTBF estimates that correspond to inefficient management of ITS resources and a lack of cross component interoperability among competing devices. In Oklahoma we conducted a study on UPS battery replacement scheduling wherein we determined that the lifetimes of UPS batteries were typically about six months longer than the times suggested within 
the manufacturer documentation. By replacing UPS batteries according to the empirically discovered MTBF estimates we greatly reduced the possibility of UPS failures at a cost proportional to the average unused energy in the discarded units. With respect to video quality monitoring systems, native solutions incorrectly depend on the strength of video signals, which fail to provide an accurate indication of the actual video quality. Overall, manufacturer suggested maintenance strategies are not optimal solutions to O\&M planning in terms of cost and quality.

We propose a low-cost distributed instrumentation system (DIS) for monitoring, identifying and diagnosing a wide variety of ITS equipment problems within our unique distributed virtual traffic management console (TMC) architecture [12]. We identify the following critical components within the state of Oklahoma ITS infrastructure,

1) 50 Portable dynamic message signs (DMS),

2) 237 Analog pan-tilt-zoom (PTZ) and IP web cameras,

3) 26 permanent dynamic message signs (DMS),

4) 61 communication cabinets,

5) 10 communication huts.

To avoid a wide range of potentially catastrophic ITS equipment failures we have developed and implemented a DIS to monitor a collection of real-time variables related to environmental conditions, physical and information technology (IT) security, information quality and energy efficiency. We have consistently employed information acquired by the DIS in all preventative maintenance decisions over the last three years, resulting in a great reduction of both maintenance cost and of unnecessary and unacceptable ITS equipment failures with significantly improved overall energy efficiency throughout the system as a whole.

\section{Distributed InStRUMENTATION SySTEM}

In [12], [13] we introduced the distributed virtual TMC deployed throughout the state of Oklahoma. The Oklahoma virtual TMC is based on a peer-to-peer network architecture where each TMC maintains an open source MySQL database that is automatically synchronized to all other visible TMCs on network. The database at each TMC node is responsible for maintaining an accurate account of all current resource allocation information that is required for managing user control of ITS equipment such as cameras and DMSs. Ideally, all TMC nodes within the ITS network are visible to one another and their databases remain perfectly synchronized. However, in the case that a critical network path becomes unavailable and the ITS network is divided into several subnetworks, the distributed nature of the system will allow each subnetwork to remain independently operational.

Due to our uniquely distributed ITS network architecture, we seek a similarly robust ITS network instrumentation strategy for monitoring and identifying potential problems within the range of ITS components visible to each network monitoring node. Each virtual TMC is a network monitoring node that employs custom software to poll and receive interrupts from all visible instrumentation devices. We propose a low-cost device that measures a large number of digital
TABLE I

SUMMARY OF LOCATIONS AND SIGNALS MONITORED BY THE DISTRIBUTED INSTRUMENTATION SYSTEM.

\begin{tabular}{|l|l|}
\hline Monitoring locations & Sensor types \\
\hline Communication Hut & Door access 1 \\
& Door access 2 \\
& Door access 3 \\
& Lo temperature \\
& Hi temperature \\
& Air Conditioner/Heater (HVAC) \# 1 \\
& Air Conditioner/Heater (HVAC) \# 2 \\
& Smoke detector \\
& Surge arrester \\
& Commercial power failure \\
\hline Cabinet & Door access \\
& Power outage \\
& UPS batteries low or bad \\
& Video synch detection \\
& Temperature \\
\hline Permanent DMS & Door access \\
& Power outage \\
& UPS batteries low or bad \\
& Temperature \\
\hline
\end{tabular}

and analog variables including temperature, battery charge, video signal integrity, security sensor status, power outage alerts, surge protection alarms and heating ventilation and air conditioning (HVAC) functionality. Table I provides a breakdown of the system monitoring needs with respect to the different types of ITS environments encountered in Oklahoma.

Fig. 1 illustrates both the distributed virtual TMC and the corresponding DIS presented in this paper. In Fig. 1, a collection of instruments and virtual TMCs are connected together by the ITS private network. The Alarm Server depicted near the center of Fig. 1 is used to transmit the information collected by the DIS to authorized users over the public internet. Our DIS operational summary that is available over the public internet is smart phone compliant as well as being compatible with most industry compliant web browser technology.

\section{A. Device Selection}

We evaluated a wide variety of commercially available development platforms for monitoring digital and analog signals in a laboratory ITS cabinet testbed. After extensive experimental testing we selected a peripheral interface controller web (PIC-web) development circuit manufactured by Olimex Ltd. over a similarly versatile ADAM-6060 module from Advantech Ltd [14]. Compared to the ADAM module, the Olimex platform is a lower cost unit that incorporates the open source MicroChip TCP/IP protocol stack (AN833), has a built in temperature sensor, and is programmable using the free MPLAB environment [14], [15]. Overall the Olimex platform provided us with a lower cost solution and 


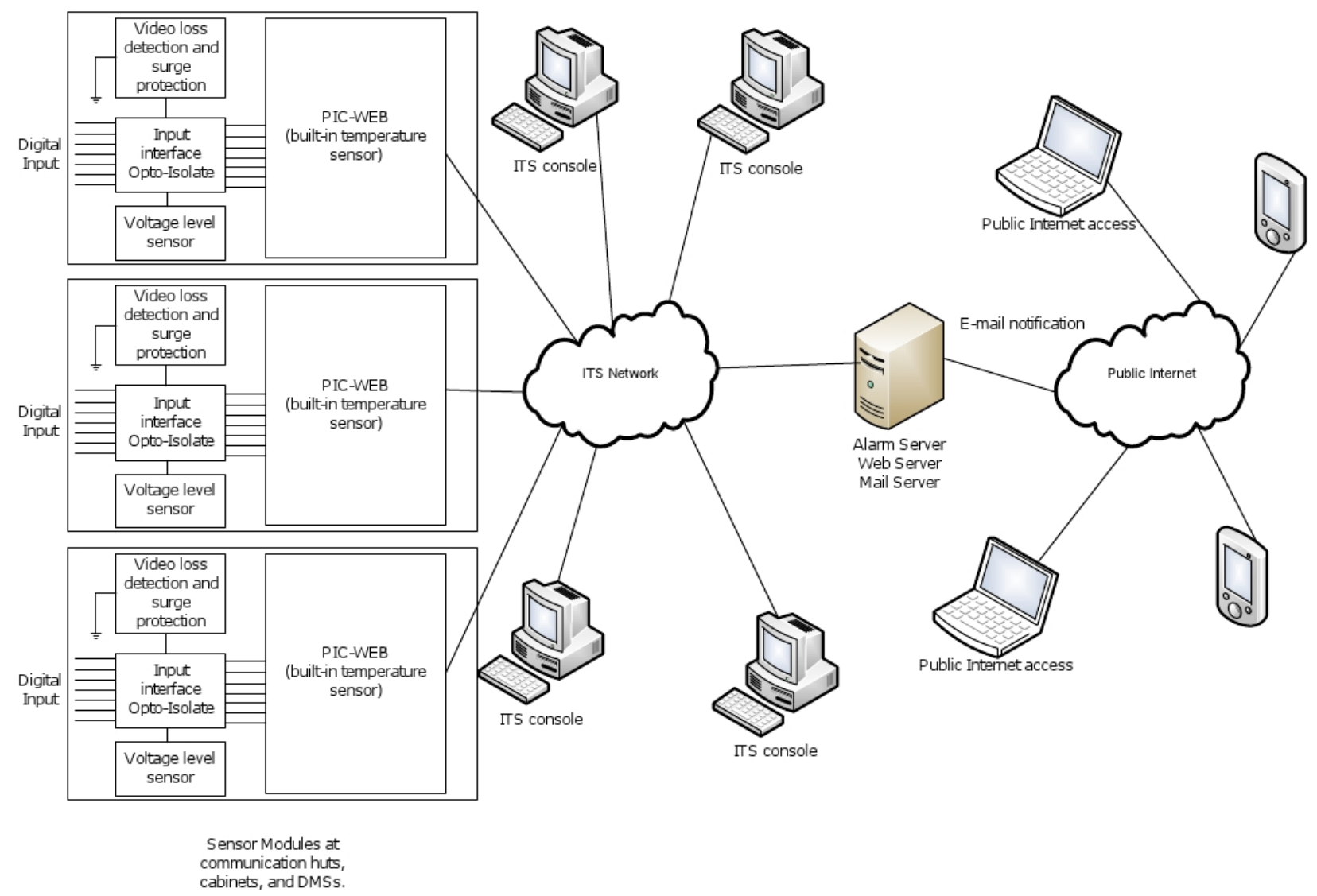

Fig. 1. Complete overview of the Oklahoma ITS virtual TMC network and the distributed instrumentation system (DIS). Examples of three instrumentation devices are shown on the left connected to the disributed TMC consoles through the ITS private network. On the right, authorized public users access an operational summary of the data gathered by the instrumentation network through a single gateway alarm server.

a reduced development time based on the inclusion of the TCP/IP implementation and a reliance on the well known MPLAB development environment.

We use the Olimex device to monitor all of the variables identified in Table I and transmit the appropriate information to and from all virtual TMCs that are visible in the network. Fig. 2 shows the Olimex instrumentation undergoing testing at the OU ITS Lab in Norman, OK. All digital signals are connected by an optical isolation interface to the input circuitry provided by the Olimex device as shown in Fig. 1 . Measurement of the analog video signal integrity and battery charge level require custom signal processing circuitry. We measure the integrity of the analog video signal based on the existence of the video synchronization signal. The analog video signal is analyzed by a custom video synchronization signal detection circuit that it is coupled to a surge protection system. To measure the battery level, a threshold is applied to the analog voltage measurement which is converted to a digital signal that indicates if the level is below the threshold. The overall cost of each Olimex-based instrument including the video synchronization detection and battery level conversion circuitry is approximately 100 USD per unit. This estimate does not include the cost of installation.

In addition to providing a robust low-cost solution for monitoring a wide variety of critical ITS devices, the Olimex platform can be easily expanded to increase the number of digital inputs or updated to reflect important firmware changes. The current instrument supports up to 8 digital inputs but this can be easily expanded to 16 digital inputs. Using in circuit serial programming (ICSP), the firmware can be upgraded without removing the peripheral interface controller (PIC) from the printed circuit board socket.

\section{B. Device Communication}

The Oklahoma DIS implementation is composed of a homogeneous collection of instrumentation devices that communicate with a similarly distributed network of virtual TMCs. The instrumentation device is a weatherproof signal monitoring unit that is based on the Olimex platform described in Section II-A. Each database on each virtual TMC maintains a list of instrumentation devices that are identified by latitude and longitude location, location description, location city, make, model, expected configuration, and a fixed IP address and port number. Each instrumentation device uses custom firmware to communicate with each virtual TMC resource management sever using the user datagram protocol (UDP).

The client firmware loaded on each device is responsible for monitoring all digital signals connected to the optically isolated input bank and communicating this information to 

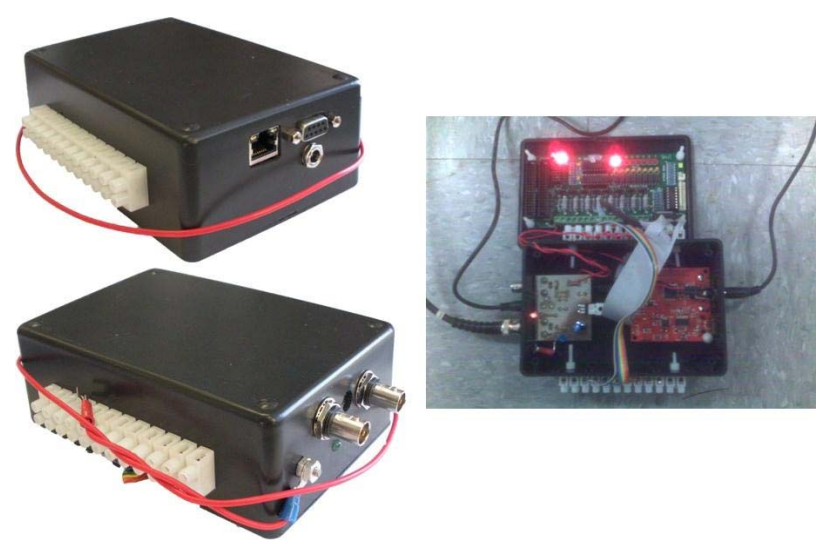

(a)

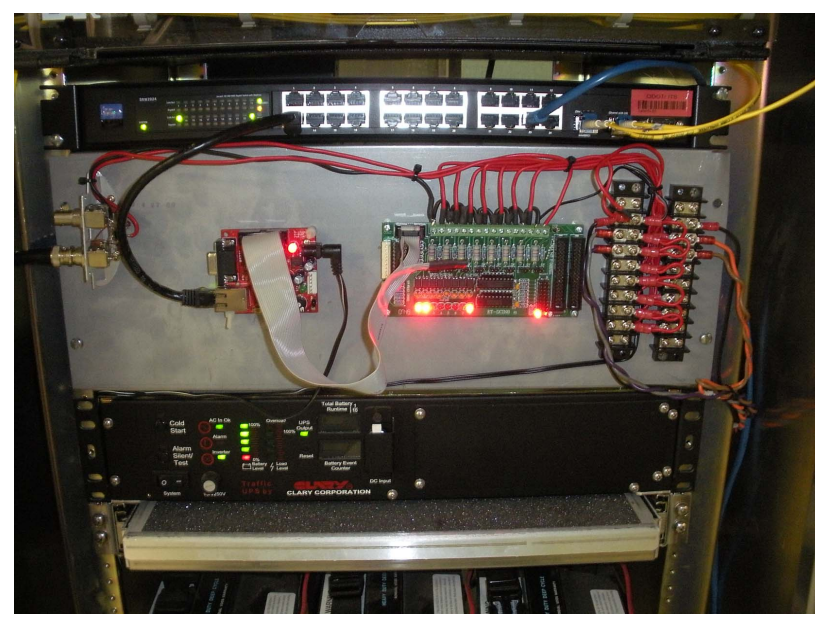

Fig. 2. A group of photos depicting the instrumentation device (a) in a low-cost weather proof project enclosure suitable for field deployment and (b) inside the communications cabinet testbed.

all relevant resource management servers. A binary representation of the status of each digital input signal is maintained within the internally available registers on the PIC microcontroller. Upon detection of a change in the status of any digital signal, the internal status register is immediately updated to reflect this change in the PIC memory. All resource management nodes within the ITS private network obtain the status of the digital signals monitored by each device by requesting the value of the internal status register. We developed the device firmware in the $\mathrm{C}$ programming language using the MicroChip MPLAB programming environment.

Each resource management server uses a custom Windows service application to communicate with all of the instrumentation devices that are visible on the ITS private network. The resource management service employs an experimental software polling technique for administration of a nonhomogeneous network of instrumentation devices. All of the monitoring devices are interrogated using manufacturer specific console applications that are dynamically loaded and executed based on the unit identification information stored in the device management database. We developed the Windows service in the Microsoft Visual Studio Development environment using the $\mathrm{C} \#$ programming language.

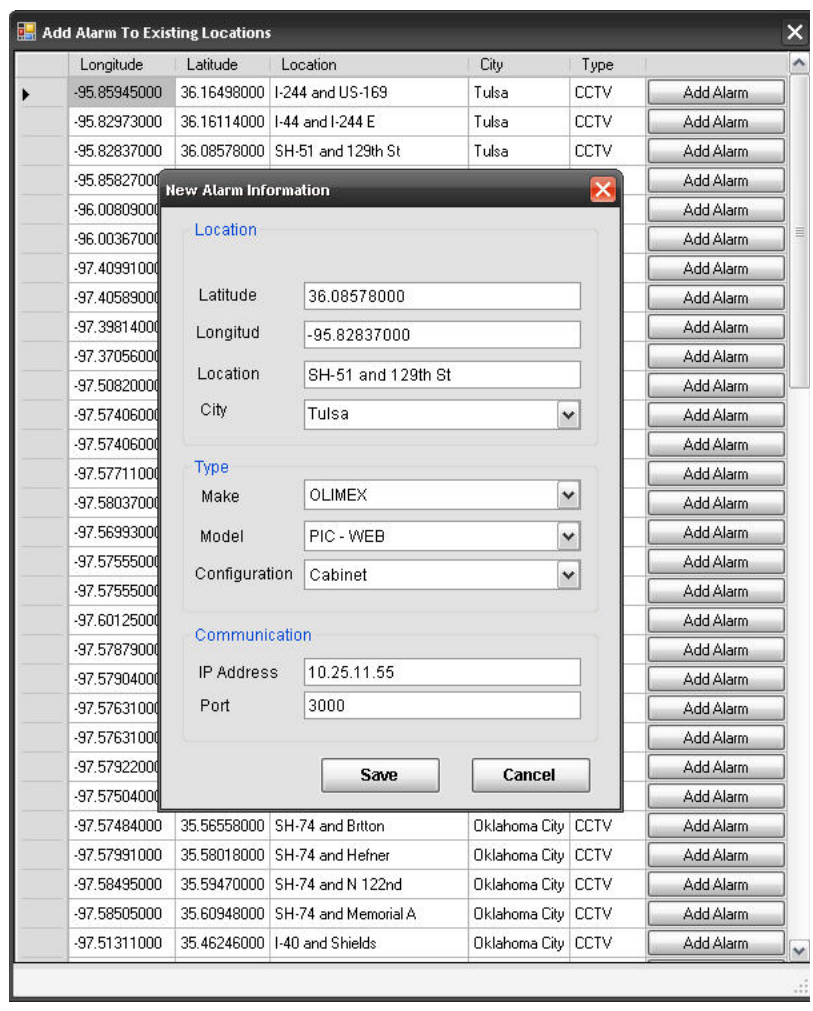

Fig. 3. Custom instrumentation device management software.

\section{USER SOFTWARE}

To support the management and distribution of information acquired with the Oklahoma DIS we developed two custom software components for managing the collection of monitoring devices and summarizing the information for ITS operators. The device resource management system was developed in Microsoft Visual Studio using the C\# programming language and an operational summary website was developed using PHP and MySQL.

The DIS resource management system is a system administration utility that is used to add, remove or edit the information associated with each instrumentation device. Fig. 3 shows the DIS resource management user interface software being used to add a new instrumentation device to a large collection of existing devices that appear in a summary table. The resource management systems can also be used to permanently remove devices from the network and to edit the information of existing devices. For example, by changing the configuration of an instrument from a communications hut to a cabinet an administrator can alter the range of variables measured by a device, given that all of the expected signals are physically connected to the unit. In other situations, a defective unit that produces too many false alarms may be removed from the network.

Fig. 4 depicts the actual website used by ITS operators, engineers and technicians to view an operational summary of critical ITS field equipment. All irregular measurements acquired by the DIS are summarized in the website using a treeview that categorizes each potential problem based on 


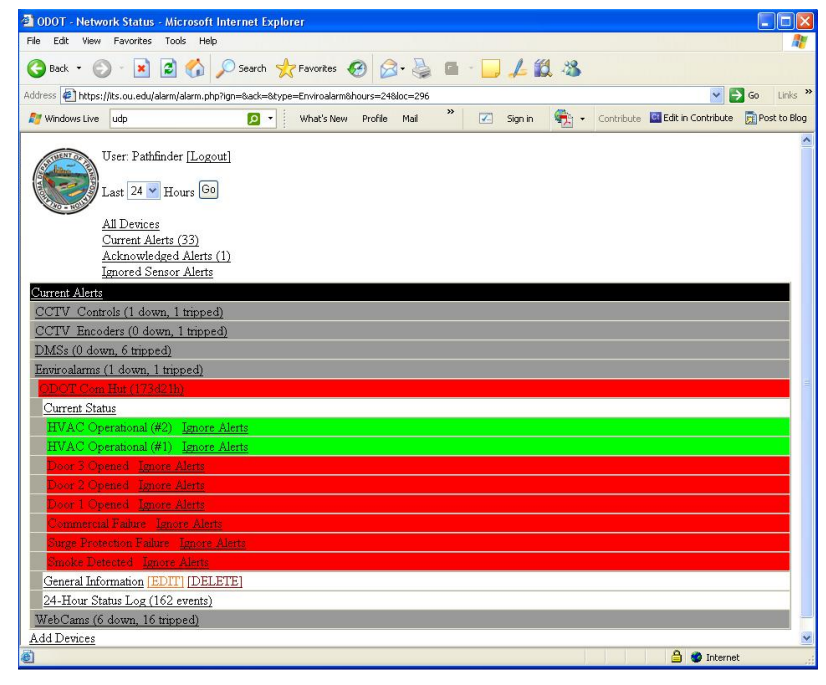

Fig. 4. Actual website used by Oklahoma ITS operators to view and check the status of ITS equipment being monitored with the distributed instrumentation system.

the type of equipment. Individual nodes within the tree can be collapsed or expanded to hide/reveal detailed descriptions of each status alert. In addition, each alert is color coded to reflect the level attention required by maintenance personnel and an alarm acknowledgement procedure is defined to ensure that every equipment problem is adequately addressed by qualified technicians.

\section{OKLAHOMA IMPLEMENTATION}

We implemented the DIS in the Oklahoma ITS private network to monitor the environmental conditions in $61 \mathrm{com}-$ munication cabinets, 10 communication huts, the integrity of 237 analog PTZ video feed and the proper functionality of 50 portable DMSs and 26 permanent DMSs. Fig. 5 shows two photographs depicting the actual installation of instrumentation devices in a communications cabinet (a) and a communications hut (b).

Each instrument was individually assembled at the OU ITS Lab in Norman Oklahoma by combining commercially available Olimex PIC-web devices with off the shelf optical isolation integrated circuits. To isolate the video synchronization signals and measure the remaining charge levels in UPS batteries we manufactured custom circuits based on our own custom designs. Lastly, each completed unit was enclosed in a low-cost weather proof container shown in 5(a).

Prior to deployment, each instrumentation device was subjected to a rigorous battery of tests conducted using a test communications cabinet and the Wireshark network protocol analysis software [16]. Each completed instrument was placed into the cabinet testbed and network monitoring software was connected in series with the communications path connecting the device to the simulated resource management server. The unit was then qualified for field service by measuring it's response to a wide range of stimuli applied to each digital signal input and measured based on an analysis of the information contained in intercepted network
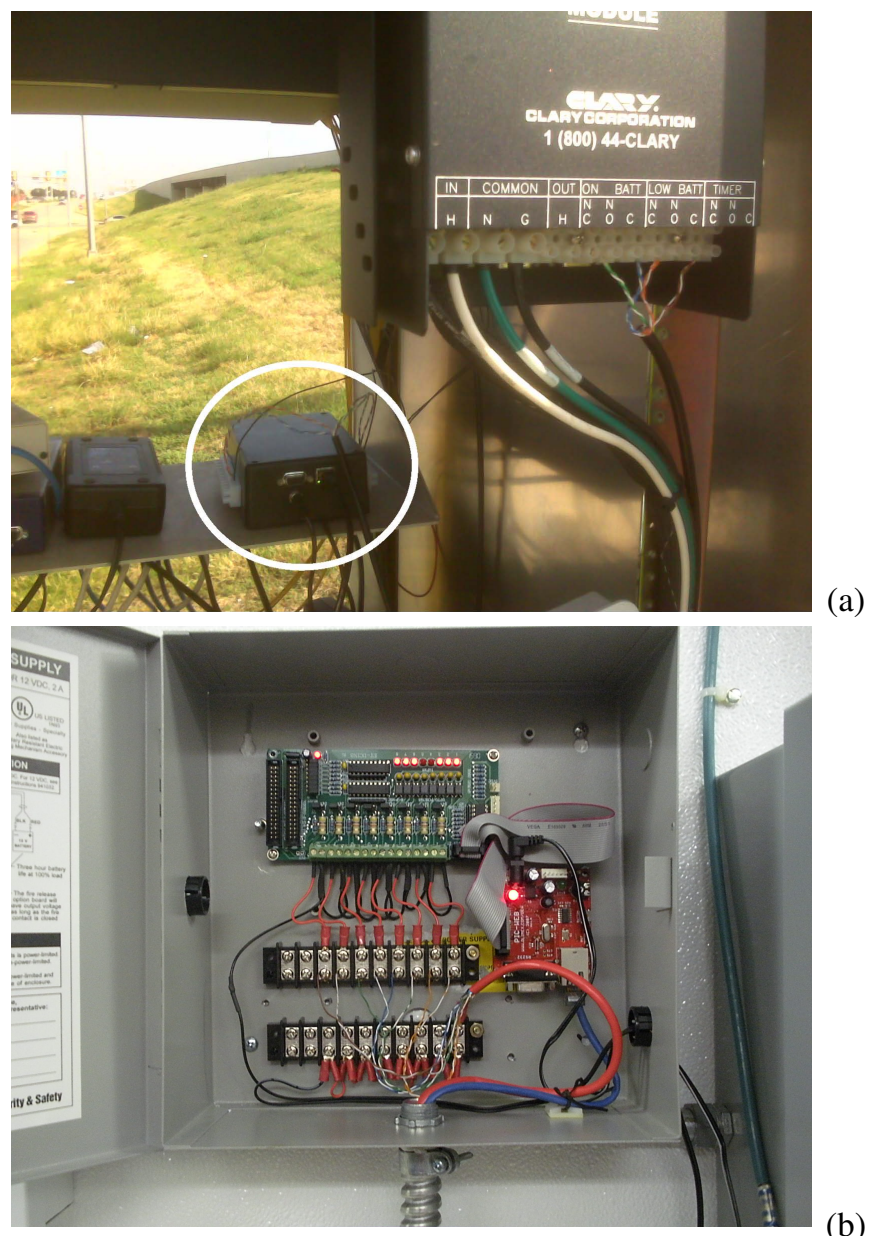

(b)

Fig. 5. Two photographs documenting (a) the instrumentation device installation at a traffic cabinet and (b) inside a communications hut.

communication packets. Fig. 6 is a screen capture of the Wireshark software taken during the testing of a single instrumentation unit [16].

A set of field ready instrumentation devices were then deployed to all of the critical ITS locations in Oklahoma and monitored by our distributed virtual TMCs. The status reports from the devices are aggregated by a single gateway computer that is accessible by authorized users from the public internet. Over the last three years, this information has been widely employed by ITS engineers and operators to assist them in their work.

\section{CONClusion}

In this paper, we presented a low-cost system for conducting surveillance on a wide variety of critical ITS components to improve preventative maintenance scheduling, reduce the possibility of equipment failures and increase the overall energy efficiency of our ITS infrastructure. Based on our existing distributed ITS architecture we designed a similarly robust monitoring system. We proposed a heterogeneous network of low-cost digital signal processing development platforms to monitor a collection of important signals acquired from environmental sensors, cameras, communica- 


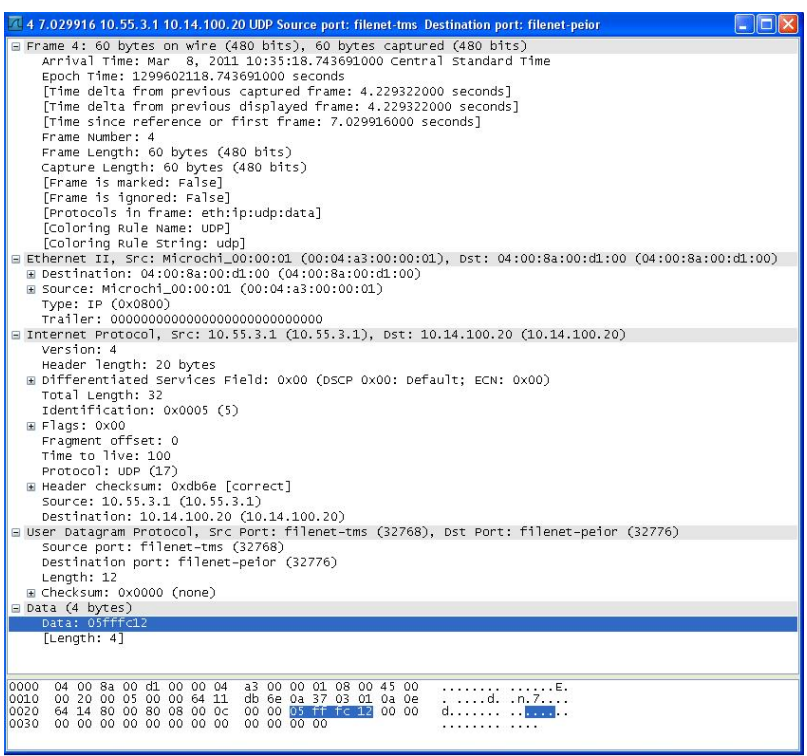

Fig. 6. Screen capture of the Wireshark software used to perform a detailed analysis of each communications packet sent or received by each instrumentation device.

tions equipment and DMSs. In addition, we presented two unique signal processing circuits for monitoring the remaining energy level of UPS batteries and measuring the integrity of video feeds based on the existence of the analog video synchronization signal.

We deployed a homogeneous DIS in Oklahoma and used it for the last three years to schedule replacement of UPS batteries, detect power failures, monitor the integrity of video feeds and ensure the operation of environmental quality control equipment. The data from the DIS are continually polled and aggregated into an executive operational summary that is available to authorized ITS personnel over the public internet 24 hours a day seven days a week. During the last several years, the data gathered by the Oklahoma DIS has contributed to a wide range of cost and energy effective O\&M planning decisions. In two specific instances, environmental data obtained by the DIS detected air conditioning failures that resulted in a significant cost savings because equipment was able to be remotely powered down before overheating occurred. In other cases, video encoder temperature measurements were used to prevent similar costs associated with heat damage to ITS equipment, battery level indicators allowed complete usage of stored energy resources and network transmission quality measurements prevented unacceptable communications failures.

\section{FUTURE WORK}

Currently, our development effort is focused on expanding the range of functionality of each PIC-web instrument to include digital output signals. By incorporating a collection of useful output control signals, the existing microcontroller network will be capable of automatically performing a wide variety of user defined maintenance tasks. In other situations, remote devices may be used over the public internet by authorized users to reboot sign controllers, cameras or network routers, resulting in a significant savings in terms of travel cost. We plan to use the information gathered by the DIS over time to develop a historical maintenance record for each piece of ITS equipment. Long term plans include the development of a website for searching maintenance records and performing data mining analysis to reveal equipment failure trends and improve O\&M planning. Lastly, we consider the potential use of ITS camera resources to document incidents of vandalism or physical security breaches at communication huts and communication cabinets. In these scenarios, an instrumentation device would automatically aim a nearby PTZ camera at an ITS facility after measuring a signal indicative of a potential security breach.

\section{REFERENCES}

[1] Missing children statistics. Visited March, 2011. [Online]. Available: http://www.missingkids.com/en_US/documents/Statistics.pdf

[2] National Center for Missing \& Exploited Children, "Analysis of amber-alert cases in 2006," U.S. Department of Justice, Tech. Rep. 2007-MC-CX-K001, 2007.

[3] — , "Analysis of amber-alert cases in 2007," U.S. Department of Justice, Tech. Rep. 2008-MC-CX-K001, 2008.

[4] — , "Analysis of amber-alert cases in 2008," U.S. Department of Justice, Tech. Rep. 2009-MC-CX-K002, 2009.

[5] — , "Analysis of amber-alert cases in 2009," U.S. Department of Justice, Tech. Rep. 2010-MC-CX-K001, 2010.

[6] P. Watson and T. Faquir, "Florida department of transportation inteliigent transportation systems maintenance - a key component to the future of an its," PBS\&J an Atkins company, Tech. Rep., May 2008.

[7] J. Walton and J. Crabtree, "Maintenance and operations plan for intelligent transportation systems in kentucky," University of Kentucky, Tech. Rep. KTC-04-14/SPR241-02-1F, Jun 2004.

[8] S. Joshua, "PECOS - ITI data models," Lee Engineering, LLC, Tech. Rep. FHWA-AZ98-464, Jun 1998.

[9] V. Carry and S. Roy, "Guidelines for transportation management system mainteance concept and plans," Federal Highway Administration, Tech. Rep. FHWA-OP-04-0111, Dec 2002.

[10] N. I. architecture team, "System engineering for intelligent transportation systems," Federal Highway Administration, Tech. Rep. FHWAHOP-07-069, Jan 2007.

[11] G. Daniels and T. Starr, "Guidelines for funding operations and maintenance of intelligent transportation systems/advanced traffic management systems," Texas Transportation Institute, Tech. Rep. 971390, Dec 1997.

[12] R. Huck, J. Havlicek, J. Sluss, Jr., and A. Stevenson, "A low-cost distributed control architecture for intelligent transportation systems deployment in the state of Oklahoma," in Proc. IEEE Int'l. Conf. Intel. Transportation Syst., Vienna, Austria, Sep. 2005, pp. 919-924.

[13] B. Kilani, E. Vorakitolan, J. Havlicek, M. Tull, and A. Stevenson, "Distributed ITS control and the Oklahoma virtual TMC," in Proc. IEEE Int'l. Conf. Intel. Transportation Syst., Saint Louis, Missouri, USA, Oct. 2009, pp. 785-790.

[14] Adam 6000 series datasheet. Visited March, 2011. [Online]. Available: http://www.advantechdirect.com/eMarketingPrograms/ L021113P1\%20Sensor/ADAM6050_51_60.DS.pdf

[15] Pic-web rev.b development board users manual. Visited March, 2011. [Online]. Available: http://www.olimex.com/dev/pdf/PIC/ PIC-WEB-B.pdf

[16] Wireshark network protocol analyzer. Visited March, 2011. [Online]. Available: http://www.wireshark.org 